\title{
Food Taboo and Its Associated Factors Among Pregnant Women in SendafaBeke Town, Oromia Regional State, Ethiopia
}

\author{
Robert Wondimu ${ }^{1, \text { *, Esubalew Tesfahun }}{ }^{2}$, Zalalem Kaba ${ }^{3}$ \\ ${ }^{1}$ SendafaBeke Town Health Office, SendafaBeke, Ethiopia \\ ${ }^{2}$ College of Health Science, Department of Public Health, DebreBehran University, DebreBehran, Ethiopia \\ ${ }^{3}$ East Wollega Zonal Health Office, WASH-NTDs Program, Nekemte, Ethiopia \\ Email address: \\ robertwondimu2021@gmail.com (R. Wondimu), esubalew.tesfahun@gmail.com(E. Tesfahun),kabazalalem@gmail.com(Z.Kaba) \\ ${ }^{*}$ Corresponding author
}

\section{To cite this article:}

Robert Wondimu, Esubalew Tesfahun, Zalalem Kaba. Food Taboo and Its Associated Factors Among Pregnant Women in SendafaBeke Town, Oromia Regional State, Ethiopia, 2019. International Journal of Science, Technology and Society. Vol. 9, No. 2, 2021 , pp. $75-86$. doi: $10.11648 /$ j.ijsts.20211002.15

Received: January 30, 2021; Accepted: March 10, 2021; Published: March 17, 2021

\begin{abstract}
Background: Dietary practices often take the form of rules stating which foods should not be eaten, that is food proscriptions or taboos. Food proscriptions are usually temporary and selective, but food taboos may have an absolute nature. Food taboos refer to the restriction of specific food as a result of social or religious customs. In many traditional societies, cultural norms and customs govern behaviors including, during critical life stages like pregnancy. Every day at least 1600 women die from the complications of pregnancy and childbirth globally. Declaring certain food taboos because they are thought to make a person sick, is also the basis for the many food taboos affecting pregnant women. Objective: To assess the prevalence of food taboo and its associated factors among pregnant women in SendafaBeke town, Oromia Regional State, Ethiopia, 2019. Methods: A community based cross-sectional study conducted from March 1, 2019 to May 17, 2019 among pregnant women who selected using systematic sampling technique. Data were collected using both a structured interviewer administered questionnaire and for qualitative, Focused Group Discussion (FGD) was conducted. The data was coded, entered into Epi data version 3.1 and exported to SPSS version 23 for data cleaning and analysis. Descriptive measures mean, median, frequency and percentage were used. Binary logistic regression was used to determine the magnitude and direction of association between a set of independent variables and the outcome variable at $\mathrm{p}<0.25$. Then those variables with $\mathrm{p}<0.25$ was selected for multivariable analysis. Finally, odds ratio with $95 \%$ confidence level was computed and p-value $<0.05$ was used to declare statistical significance. Results: More than half (55.3\%) of total pregnant mothers encountered for at least for one food item. Food items avoided were, milk and milk products (26.9\%), eggs (20.23\%), linseed (17.86\%), fatty meats (15.07\%), fruits (9.52\%), Honey $(6.74 \%)$ and vegetables $(3.57 \%)$. Reasons mentioned for avoidance of this food item; fear of fat baby, fear of abnormality baby, fear of abortion, plastered on fetal head and food flavor. Conclusion: This study revealed that food taboos and eating behavior during pregnancy like aversion, skipping and no use additional meal and occupation of husbands and educational status shows that significant association with food taboo affects more than half of pregnant mothers due to beliefs in old unscientific tales.
\end{abstract}

Keywords: Food Taboo, Pregnant Women, SendafaBeke, Oromia

\section{Introduction}

Dietary practices often take the form of rules stating which foods should not be eaten, that is food proscriptions or taboos [1]. Food proscriptions are usually temporary and selective, but food taboos may have an absolute nature. Some well- known examples of absolute food taboos are religious taboos, such as the pork taboo among Muslims and the Hindu beef taboo. In most cultures; women and children are considered to be particularly vulnerable during pregnancy, childbirth and the postpartum period. These stages are therefore frequently characterized by various beliefs and practices designed to 
protect the physical and spiritual health of the mother and infant by determining amongst others when, what and how the mother and infant eat and when what type of health care is needed [2].

During pregnancy, the growing baby receives all its nourishment from the mother's body. When stores of carbohydrates, proteins, fats, vitamins, minerals and other nutrients are low, a woman's body will drain them to support the growth and development of the baby. If reserves are not sufficiently restored through healthy eating, the mother can become severely malnourished, and this in turn can lead to depression, exhaustion and many other serious health complications [3]. Every day at least 1,600 women die from the complications of pregnancy and childbirth globally. This means that at a minimum 585,000 women die each year. The great majority of the deaths $(99 \%)$ take place in developing countries. Each year over 50 million women experience pregnancy related complications, many of which lead to long term illness or disability. Complications of pregnancy and childbirth are the leading cause of death and disability for women in developing country aged 15-49. Women's lifetime risk of dying from pregnancy in Africa is 1 in 16-20 while it is 1 in 1400 in Europe and 1 in 3700 in North America [4].

In many countries, women have to deal with food restrictions during their pregnancy or lactation. Many of these food taboos are related to animal-source foods or fruits and vegetables. Restriction of these foods during pregnancy and lactation can be very harmful for both the mother and fetus, as essentials nutrients such as protein and vitamins are lacking in the diet $[5,6]$. Food taboos are a common practice of prohibiting certain food items for pregnant and/or lactating women or girls in general. Children, especially girls, are most vulnerable to this practice. Foods that are good sources of energy and protein are not allowed to be consumed by pregnant women for reasons such as difficult and prolonged labor due to fears of a large baby. Similarly sources of vitamins and minerals are restricted during pregnancy mainly due to the fear of offensive discharges during delivery and skin diseases on the body [3].

Pregnant women avoid specific foods due to several reasons. Some pregnant women avoid foods as a result of strong dislike (aversion) developed following pregnancy while other women avoid on medical grounds. In developing country however a substantial number of pregnant women avoid specific foods due to cultural beliefs or impositions. The practice of avoidance of foods due to cultural food beliefs is referred to as food taboos. Cultural food restriction during pregnancy is common practice, particularly in developing country. High prevalence of food taboo practice was reported in several areas of the world. Food taboos have been identified as one of the factors contributing to maternal under nutrition in pregnancy, especially in developing country [7].

According to the study in Malaysia, the prevalence rate of pregnant women adhering to specific food taboos during their pregnancy was $70.2 \%$, of which, $18.3 \%$ avoided eating at least one food item [8].
In Ethiopia few studies have focused on dietary habits of pregnant women and the intake of specific nutrients especially micronutrient and the effect on pregnancy outcome and complication. Evidence about Ethiopian women's dietary composition and habits during pregnancy and nutrient intake is however lacking [9]. More than a third of child deaths and $11 \%$ of the total disease burden worldwide are due to maternal and child under nutrition and More than 3.5 million women and children under age five in developing countries die each year due to the underlying cause of under nutrition and Ethiopia is one of the developing country with very high burden of maternal and child under nutrition [6].

Maternal dietary habits, poor dietary pattern such as food taboo and poor nutritional status of women during pregnancy is one of the major causes of anemia in pregnancy [10]. The United Nations estimated that most of the 8.41 million chronically hungry people in developing countries are women and children and this infringes on their fundamental human rights [8]. The food consumption studies during pregnancy and lactation in sub-Saharan Africa Countries show that nutrient intakes are low in the range of 1400- 2000 Kcal of energy $25-50 \mathrm{Kg}$ protein while vitamins and minerals intake are extremely low [11].

Well-being of mother and the new born infant is greatly determined by the nutrition of the expectant mother during pregnancy and it further influences the health of the child during childhood and adulthood. Cravings and aversions, which refer to a strong desire and strong dislike for certain food respectively, are common during pregnancy with complications such as nausea and Vomiting [12]. Many Women in Africa suffer from chronic under nutrition and micronutrient deficiencies and fail to gain enough weight during pregnancy. The consequences of malnutrition for mothers include increased risk of death, illness, and complications during pregnancy and childbirth, greater susceptibility to infection, reduced activity levels, and lower productivity [13].

Severe food avoidance during pregnancy might deplete the body of important nutrients which can adversely affect pregnancy and birth out comes. Evidence showed that the prevalence of food taboo in Shashamane town (49.8\%), In Hadiya (27\%) and In Nigeria (16.1\%) [14, 12, 11].

Food taboos recorded all over the world differ only in type and characteristics. The more frequent taboo was related to the simultaneous consumption of milk and fruits, such as mango, orange, pineapple and nuts. They also considered eating eggs and fruits together harmful, as well as a combination of meat with fish [3]. Poor nutrition that results from inadequate dietary intake is associated with a range of social, economic and cultural factors. Declaring certain foods taboo because they are thought to make a person sick, is also the basis for the many food taboos affecting pregnant women [15].

Food habits share the adaptive nature of culture, religion and traditional knowledge: "food Habits, whereas they are basically stable and predictable they are, paradoxically, at the same time undergoing constant and continuous change. Change occurs over time because of ecological and economic 
changes leading to altered availability discover or innovations of foods and diffusion or borrowing of food habits from others [2]. The major problems the women had during pregnancy in relation to feeding are unavailability of foods they like to eat; food scarcity. Lack of appetite, heartburn, constipation, vomiting, insufficient money; and lack of knowledge of nutritious foods. In order to overcome the problem of lack of appetite and constipation they averted some type of food in their pregnancy life [8].

Inadequate and/or inappropriate knowledge and discriminatory attitudes limit household access to actual resources because political, cultural, religious, economic and social systems including status of women limit the utilization of potential resources [16]. Although food taboo is common among pregnant women in developing countries including Ethiopia, the magnitude of the problem and its predictors were not well documented in Ethiopian in general and in the study area in particular. Malnutrition is a significant health problem affecting pregnant women in Ethiopia and has serious consequences for pregnancy and birth outcomes [11, 7]. This study is going to assess food taboo among the pregnant women and its associated factors in the context of the study area. Therefore this study identify that the magnitude of Food taboo among pregnant women and its associated factor.

\section{Methods and Materials}

\subsection{Study Area}

The study was conducted in SendafaBeke Town one of the Addis Ababa Zuriya (around) Special Zone of Oromia Regional state, Ethiopia. SendafaBeke Town has 56,350 total populations out of which 28,175 are male and the rest 28,175 are females and 10,914 annual expected pregnancies. SendafaBeke Town have one district hospital, two health center, one health post, two high school, one preparatory school, four primary school and one technical and vocational training collage. It is $38 \mathrm{KM}$ far from Addis Ababa (capital of the nation) in the North and 92KM from DebreBehran; the capital city of the North Shoa Zone.

\subsection{Study Design and Period}

Community based cross-sectional quantitative study supported by qualitative approach was used. The study was undertaken from March 1, 2019 to May 17, 2019.

\subsection{Population}

\subsubsection{Source Population}

For quantitative:-all women who are pregnant at the time of the study who live in the study area. For qualitative:-all model house hold during study period.

\subsubsection{Study Population}

For quantitative data: selected pregnant women who live in SendafaBeke Town at the time of the survey and fulfill the inclusion criteria. For qualitative data: - selected pregnant women for FGD from model house hold who fulfill inclusion criteria.

\subsection{Sample Size Determination}

For quantitative:-a single population proportion was used to determine sample size based on the assumption of $49.8 \%$ prevalence of food taboo [17], expected margin of error (d) 0.05 at $95 \%$ confidence level $(\mathrm{Za} / 2)$ for quantitative study. Considering non-response rate of $10 \%$, the sample size was computed to be as follows:-

$$
\begin{gathered}
\mathrm{nf}=\frac{\left(N\left(Z_{1}-\alpha / 2\right)^{2}\right) P(1-P)}{d^{2}(N-1)+\left(z_{1}{ }^{\alpha} / 2\right)^{2} P(1-P)} \\
\mathrm{nf}=\frac{\left(914(-0.96)^{2}\right) 0.498(1-0.498)}{0.05^{2}(914-1)+(-0.96)^{2} 0.498(1-0.498)} \\
\mathrm{n}=384
\end{gathered}
$$

$\mathrm{n}=$ using this formula and adding $10 \%$ non-response rate the sample size is 422 .

Where; $\mathrm{Z}=$ Confidence level, $\mathrm{N}=$ total pregnant women in that town, $d=$ margin of error to be tolerated, and $p=$ prevalence of existing food taboo

For qualitative study focused group discussion of 4 groups 30 (persons) in which each group have 6-8 participants was conducted.

\subsection{Sampling Procedures}

All pregnant mothers in the town was listed down by taking their list from town health extension workers and the study individuals was selected using systematic sampling technique.

Sampling for qualitative study

For qualitative study based on the inclusion criteria the participants was selected in collaboration with health extension workers and Kebele administration.

\subsection{Inclusion and Exclusion Criteria}

\subsubsection{Inclusion Criteria}

Pregnant women aged 19 to 49 years who were healthy at the time of data collection were included in the study. Pregnant women who lived at list for six and more months in the study area were included. Pregnant women who following antenatal care (ANC) at health post (HP) and those who are with visible pregnancy (second and third trimester) but not following ANC service from HP were included. Pregnant women who are in the first trimester and no visible pregnancy but following ANC service from health post were included.

\subsubsection{Exclusion Criteria}

Pregnant women who are sick at the time of data collection were excluded. Unhealthy pregnant women who have infectious disease were excluded. 


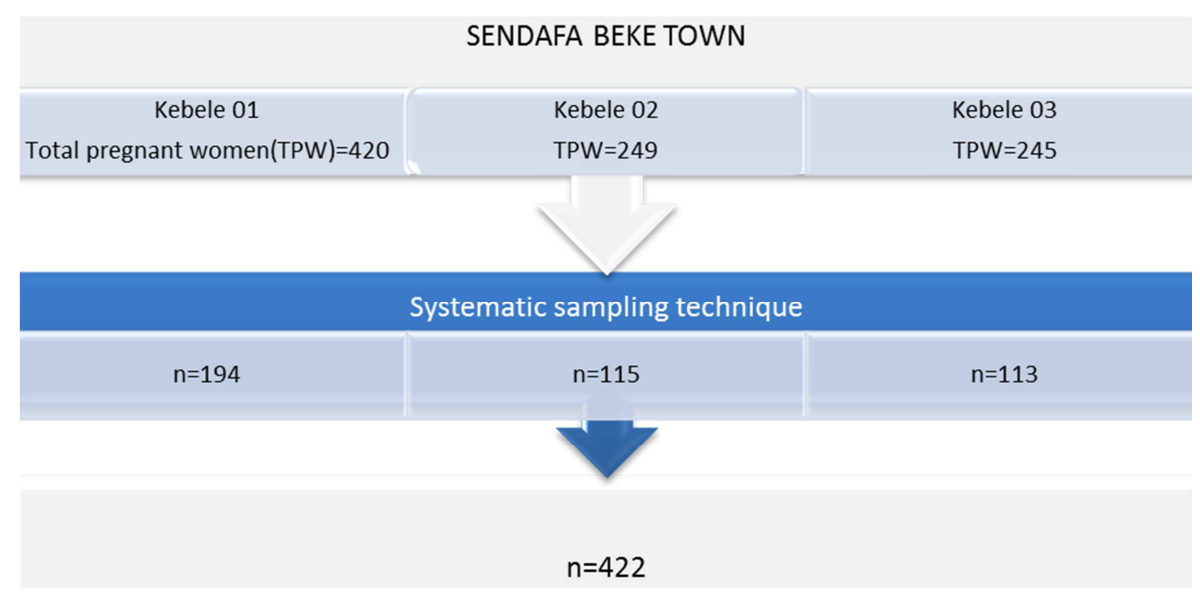

Figure 1. Diagrammatic representation of sampling procedure for Food taboo and its associated Factor among Pregnant women in SendafaBeke town, Oromia Regional State, Ethiopia, 2019.

\subsection{Variables}

\subsubsection{Dependent Variable}

Food taboo during pregnancy

\subsubsection{Independent Variables}

Socio-demographic and/or economic status:- Income, religion, age, education, marital status, Culture and belief

Individual factor:- Occupation, Family planning utilization, ANC service utilization

Environmental factor:-Production of cereals and farm land ownership

Factor related to pregnancy:- Food aversion, food craving

Eating Behavior:-Pica consumption, skipping meal

\subsection{Operational Definition}

Food taboo:-is a common practice of prohibiting certain food items for pregnant women or girls in general [3].

Vegetable and fruit serving:-recommended number of serving of vegetable and fruit by world health organization (WHO) is five serving of vegetable per day per pregnant women.

Two and less serving of vegetable per day:-less than recommended vegetable consumption

Three-four servings of vegetables per day:-slightly good

Five and more servings of vegetable per day:-very good

Feeding:-in this case it is used similarly as eating.

Regular Meal serving per day of pregnant women:

Once:-very low eating habit

Twice:-low eating habit

Three:-good eating habit

Four and more:-is very good eating habit

Additional food serving per day:-

Once:-very low eating habit

Twice:-low eating habit

Three:-good eating habit

Four and more:-is very good eating habit

Coffee consumption per coffee ceremony:-

One cup:-low

Two cup:-medium

Three cup:-high

\subsection{Data Collection Methods and Tools}

Data were collected by face to face interview using pretested structured questionnaire which was adapted from other studies. Focused Group Discussion (FGD) guide line was used which is adapted from world health organization (WHO) safe child birth check list collaboration.

\subsection{Data Collection Procedure}

Data collectors who were fluent in regional language (Afan Oromo) and who are qualified at least with diploma in nursing or public health collected the data after they take training on the data collection instrument. Bachelor of Science professional was assigned as a supervisor for data collection process. Data for qualitative was collected by FGD guide line and record was kept on paper and tape recording was conducted by taking consent from participants.

\subsection{Data Processing and Analysis}

Quantitative data analyzed at two levels. First, bivariate analysis was performed to determine the differentials of Food taboo by explanatory variables. Pearson's chi-square test of independence was performed to test the existence of significant association between food taboo and selected risk factors. Then, the significant variables ( $p$-value $<0.25$ ) observed in bivariate analysis was subsequently included in multivariate analysis. Logistic regression model was applied to examine independent associations between explanatory variables and a binary dependent variable. Data were entered in to Epidata version 3.01 and explored and analyzed Using SPSS version 23 software. A multivariable logistic regression analysis was used to isolate the independent effect of independent variables on dependent variable. The qualitative data were transcribed verbatim into to English language and carefully read. Then themes were developed which was used for coding and analyzed based on thematic framework. Then, the results were presented using narratives using well said verbatim as illustrations. 


\subsection{Data Quality Assurance}

For quantitative study to ensure quality of data the questionnaire was pretested. Data collectors who have at least diploma level training in nursing or public health were selected and training was given. Supervision, checking consistency and completeness, data cleaning was undertaken. For the quantitative study in addition to the above activities record of raw data were kept on paper as well as by tape recording the transcribing it on to paper.

\subsection{Ethical Consideration}

Prior to starting the study, the final proposal was submitted to DebreBehran University Review Board for ethical approval. Then ethical clearance was taken from DebreBehran University. After permission was taken from Addis Ababa Zuria Zonal Health department. All participants have informed the right not to answer any questions or all questions if they want. Respect and full confidentiality of the study participant's response was granted since only the investigator had access to the data. Then information was collected after securing oral consent from study participant. Permission was first sought and obtained from the kebele administrate after explaining the purpose of the study.

\section{Results}

\subsection{Socio Demographic Characteristics}

A total of 407 pregnant women were participated in the current study, which yield a response rate of $96.45 \%$. The study participating with mean age of 27 years $(27+4.67 \mathrm{SD})$, $209(51.4 .1 \%)$ were Oromo, $196(48.2 \%)$ were orthodox Christian by their religion, $343(84.3 \%)$ married those who living together, 135 (33.2\%) were primary school (1-8), 214 (52.3\%) Housewife, 164 (40.3\%) merchant husbands, 365 (91.48\%) their monthly income were categorized more than $\geq 2001,365$ (91.48\%) were 4-6 family size and $168(41.3 \%)$ two parity history [Table 1].

\subsection{Status of Pregnant Women Associated with Fasting, Food Aversion and Food Craving}

From 407 pregnant mother interviewed; 152 (37.3\%) fasting during their current pregnant, and $108(71.05 \%)$ of them were fasting every Wednesday and Friday [Figure 2].
Table 1. Socio-demographic characteristics of Pregnant women in SendafaBeke town, Oromia Regional State, Ethiopia, June 2019 ( $n=407)$.

\begin{tabular}{|c|c|c|}
\hline Variables & Frequency (N) & Percent (\%) \\
\hline \multicolumn{3}{|l|}{ Age group (in years) } \\
\hline 19 & 14 & 3.4 \\
\hline $20-24$ & 119 & 29.2 \\
\hline $25-29$ & 153 & 37.6 \\
\hline $30-34$ & 103 & 25.3 \\
\hline$\geq 35$ & 18 & 4.4 \\
\hline \multicolumn{3}{|l|}{ Ethnicity } \\
\hline Oromo & 209 & 51.4 \\
\hline Amhara & 108 & 26.5 \\
\hline Gurage & 38 & 9.3 \\
\hline Tigre & 49 & 12.0 \\
\hline Others & 3 & 0.7 \\
\hline \multicolumn{3}{|l|}{ Religion } \\
\hline Orthodox & 196 & 48.2 \\
\hline Protestant & 112 & 27.5 \\
\hline Muslims & 60 & 14.7 \\
\hline Wakefata & 39 & 9.6 \\
\hline \multicolumn{3}{|l|}{ Educational status } \\
\hline unable to read and write & 61 & 15 \\
\hline able to read and write & 91 & 22.4 \\
\hline Primary [1-8] & 135 & 33.2 \\
\hline Secondary [9-12] & 61 & 15 \\
\hline College diploma and above & 59 & 14.5 \\
\hline \multicolumn{3}{|l|}{ Occupation of mother } \\
\hline House wife & 214 & 52.3 \\
\hline Govt. employee & 62 & 15.2 \\
\hline Merchant & 127 & 31.2 \\
\hline Farmer & 2 & 0.5 \\
\hline Other & 2 & 0.5 \\
\hline \multicolumn{3}{|l|}{ Occupation of Husband } \\
\hline Govt. employee & 157 & 38.6 \\
\hline Merchant & 164 & 40.3 \\
\hline Daily Laborer & 4 & 1.0 \\
\hline Farmer & 40 & 9.8 \\
\hline Driver & 37 & 9.1 \\
\hline \multicolumn{3}{|l|}{ Family Size } \\
\hline $1-3$ & 132 & 32.4 \\
\hline $4-6$ & 235 & 57.7 \\
\hline$\geq 7$ & 40 & 9.8 \\
\hline \multicolumn{3}{|l|}{ Number of Parity } \\
\hline No birth before & 75 & 18.4 \\
\hline one & 83 & 20.4 \\
\hline Two & 168 & 41.3 \\
\hline $3-6$ & 81 & 19.9 \\
\hline
\end{tabular}



Figure 2. Pregnant women fasting during their pregnancy in SendafaBeke town, Oromia Regional State, Ethiopia, June, 2019. 
Of all 407 interviewed pregnant mother; 252 (61.9\%) were averted at least one item of foods. Among them 68 (26.9\%), 51 (20.23\%), 45 (17.86\%), 38 (15.07), 24 (9.52\%), 17 (6.74\%) and 9 (3.57\%) averted Milk, egg, linseed, fatty meat, fruits, honey and vegetables respectively [Figure 3].

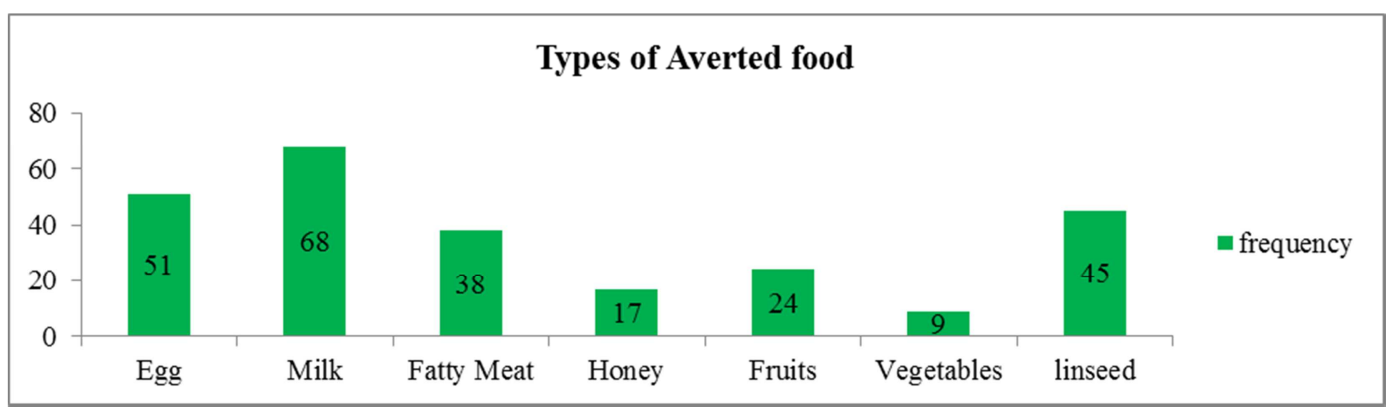

Figure 3. Types of averted food in pregnant women during their pregnancy in SendafaBeke town, Oromia Regional State, Ethiopia, June, 2019.

Reasons for aversion was highly leaded by fear of fat baby, 101 (40.08\%) and followed by fear of abnormality baby 55 (21.82) and fear of abortion 47 (18.65\%) [Figure 4].



Figure 4. Reasons for Averting food among pregnant women during their pregnancy in SendafaBeke town, Oromia Regional State, Ethiopia, June, 2019.

Among 252 pregnant women who averted food were 248 (98.4\%) during first trimester and $3(1.19 \%)$ and $1(0.39 \%)$ during second and third trimester respectively [Figure 5].



Figure 5. Time of Averting food among pregnant women during their pregnancy in SendafaBeke town, Oromia Regional State, Ethiopia, June, 2019.

Of all 407 participants $210(51.6 \%)$ craved foods during their pregnant and craved $150(71.43 \%)$ meat followed by 53 (25.24\%) bread [Table 2].

Table 2. Craving practice and types of Craved foods by pregnant women in SendafaBeke town, Oromia Regional State, Ethiopia, June, 2019.

\begin{tabular}{lll}
\hline Variables & Frequency $(\mathbf{N})$ & Percent $(\mathbf{\%})$ \\
\hline Craving & & \\
YES & 210 & 51.6 \\
NO & 197 & 48.4 \\
Food Type & 3 & 1.43 \\
Eggs & 150 & 71.43 \\
Meat & 53 & 25.24 \\
Bread & 4 & 1.90 \\
Others & & \\
\hline
\end{tabular}


From all pregnant women 108 (51.43\%) were craved foods because of taste of foods, 90 (42.85\%), $11(5.24 \%) 1(0.48 \%)$ reasoned for food flavor, personal interest and because of advice from others respectively [Figure 6].

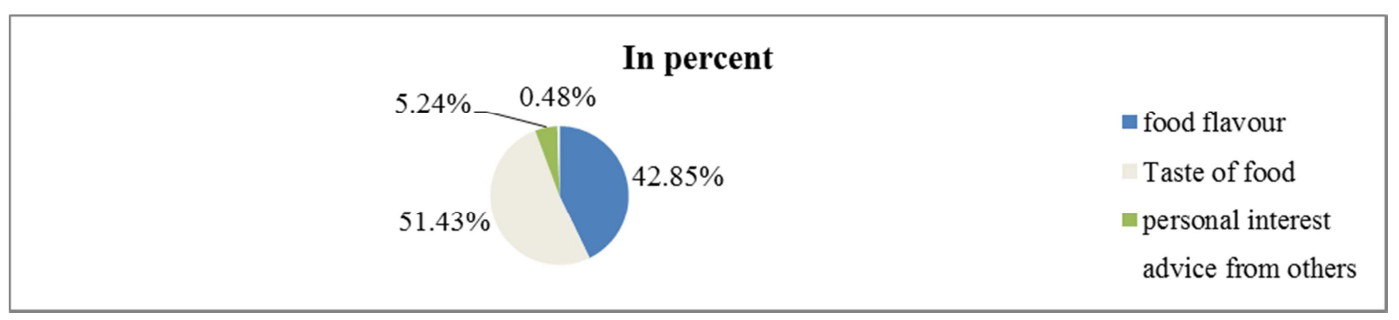

Figure 6. Reasons why pregnant women craved foods in SendafaBeke town, Oromia Regional State, Ethiopia, June, 2019.

\subsection{Types of Food Taboo, Vegetable and Fruits Consumption Practice}

Among 407 pregnant women respond 105 (25.8\%) Any white color food items are considered in their community and followed by 90 (22.1\%), 83 (20.4\%) and 79 (19.4\%) vegetables, linseed and egg respectively [Table 3].

Table 3. Types of Foods Considered as taboo by of pregnant women in SendafaBeke town, Oromia Regional State, Ethiopia, June, 2019.

\begin{tabular}{|c|c|c|}
\hline Types of foods & Frequency & Percentage \\
\hline Fatty Food & 3 & 0.7 \\
\hline Milk product & 40 & 9.8 \\
\hline Egg & 79 & 19.4 \\
\hline Any white color & 105 & 25.8 \\
\hline Vegetable & 90 & 22.1 \\
\hline Fruits & 7 & 1.7 \\
\hline
\end{tabular}

About $225(55.3 \%)$ respondent were not Eat one food type during their pregnancy that they respond as food taboo in community [Figure 7].

\begin{tabular}{|c|c|}
\hline \multicolumn{2}{|c|}{ Satus Practice of tabooed food } \\
\hline $44.70 \%$ & $\begin{array}{c}\text { YES } \\
\text { NO }\end{array}$ \\
\hline
\end{tabular}

Figure 7. Status of practicing taboo food categorization in community of pregnant women in SendafaBeke town, Oromia Regional State, Ethiopia, June, 2019.

From 407 respondent, 207 (50.9\%) not use vegetables and fruits during their pregnant. All of them who had practicing eating Vegetables and fruits had bought from market and once time per day. About 200 (49.1\%) of them uses vegetables and fruits sometimes during their pregnancy.

\subsection{Types of Production They Produce and Eat Commonly}

About $97(23.8 \%)$ respondent have land farm in rural area. Among them $86(88.66 \%)$ Producing combination of products and $6(6.18 \%)$ of them only produce teff. From product they produce $79(81.44 \%)$ used for both market and at home level [Table 4].

Table 4. Status of Having Land Farm, Types and purpose of product pregnant women in SendafaBeke town, Oromia Regional State, Ethiopia, June, 2019.

\begin{tabular}{llll}
\hline Category & & Frequency & Percentage \\
\hline Land Farming From Rural area & Yes & 97 & 23.8 \\
& No & 310 & 76.2 \\
& Teff & 6 & 6.18 \\
Types of Production & Bean & 1 & 1.03 \\
& Combination & 86 & 48.66 \\
& Others & 4 & 4.1 \\
Production purposed (used) & For market & 2 & 2.06 \\
& For home use & 16 & 16.49 \\
\hline
\end{tabular}


From respondent $302(74.85 \%)$ were used Teff, and $64(15.7 \%)$ were used wheat commonly in their house hold level [Figure $8]$.

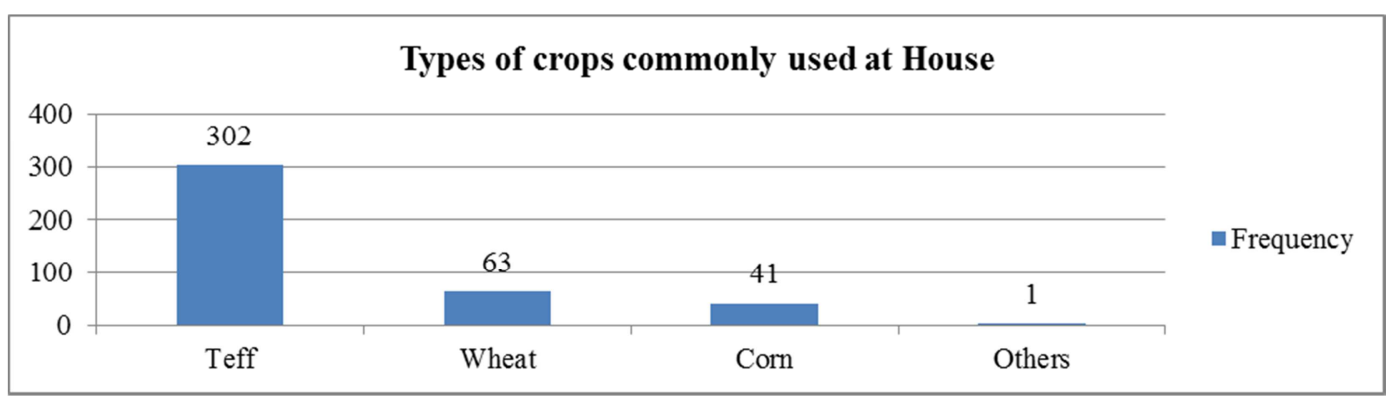

Figure 8. Types of Crops commonly used in house at pregnant women in SendafaBeke town, Oromia Regional State, Ethiopia, June, 2019.

\subsection{Time and Frequency of Eating During Pregnancy}

Among 407 study participants 260 (63.9\%) ate together with family and 261 (64.1\%) eat three times per day [Table 5].

Table 5. Time when pregnant mothers eat meal and its frequency per day in SendafaBeke town, Oromia Regional State, Ethiopia, June, 2019.

\begin{tabular}{|c|c|c|c|}
\hline Category & & Frequency & Percentage \\
\hline \multirow{4}{*}{ When Mothers eat Meal } & Before family eat & 5 & 1.2 \\
\hline & After family ate & 4 & 1.0 \\
\hline & With Husband & 138 & 33.9 \\
\hline & With family together & 260 & 63.9 \\
\hline \multirow{2}{*}{$\begin{array}{l}\text { Frequency of mothers' meal per } \\
\text { day. }\end{array}$} & Twice & 3 & 0.7 \\
\hline & Three times & 261 & 64.1 \\
\hline
\end{tabular}

From 407 study participant 122 (30\%) eat additional meal and among them 105 (86.07\%) add one times per day [Figure 9].

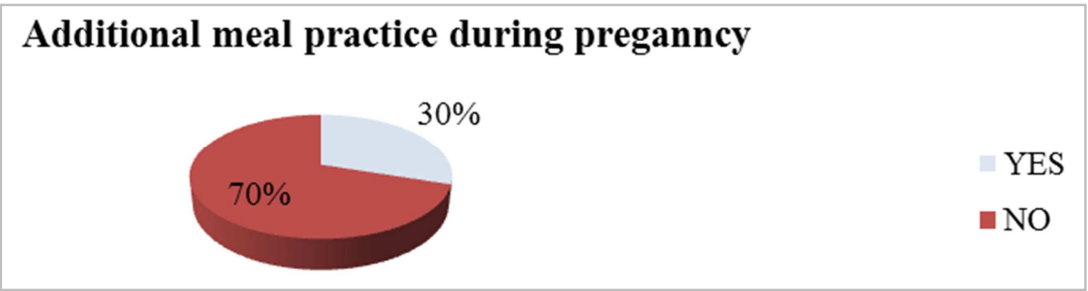

Figure 9. Practice of additional meal practice to pregnant women in SendafaBeke town, Oromia Regional State, Ethiopia, June, 2019.

From those who had a practice of additional meal during their pregnancy $105(86.07 \%)$ of them ate one times per day [Figure 10].

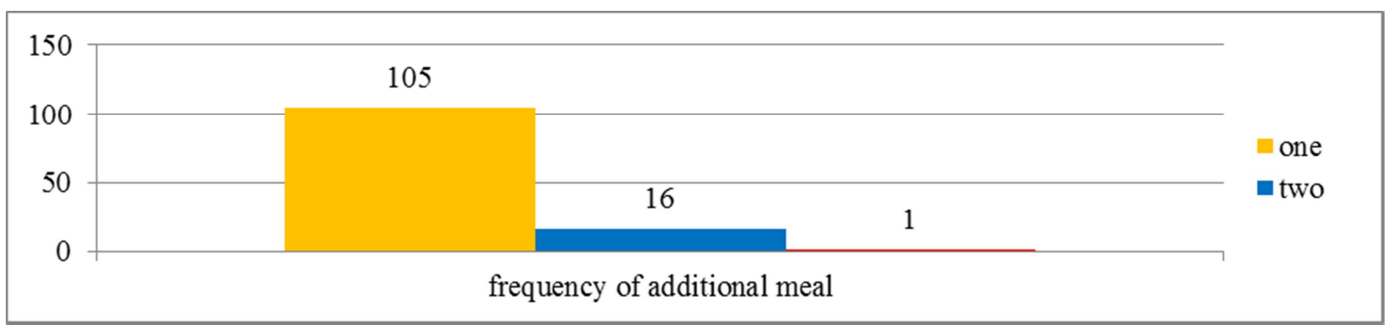

Figure 10. Frequency of additional meal practice to pregnant women in SendafaBeke town, Oromia Regional State, Ethiopia, June, 2019.

Among 285 (70\%) pregnant women did not eat additional meal through their pregnancy time and they reason out Fear of delivery difficulty counts $141(49.47 \%)$ and followed by fear of weight gain 132 (46.32\%) and 7 (2.46\%) no enough food in home and others [Figure 11]. 


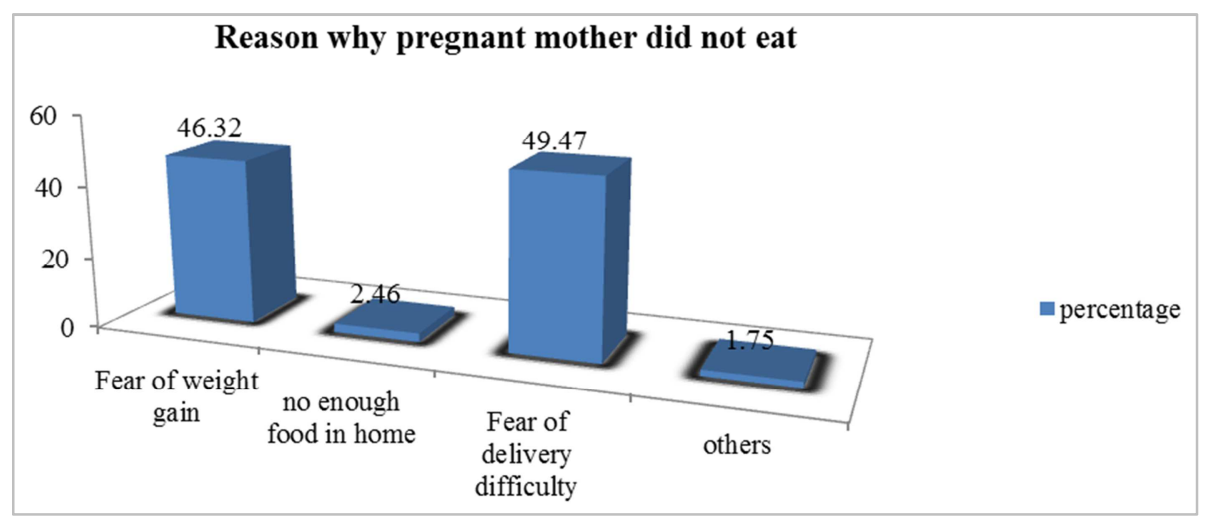

Figure 11. Reasons why did not eat additional meal pregnant women in SendafaBeke town, Oromia Regional State, Ethiopia, June, 2019.

Among 407 pregnant women 172 (42.3\%) skip foods some times 98 (56.98\%) during breakfast 88 (51.16\%) due to strong dislike $39(22.67 \%)$ [Table 6].

Table 6. Skip of practicing taboo food categorization in community of pregnant women in SendafaBeke town, Oromia Regional State, Ethiopia, June, 2019 $(n=407)$.

\begin{tabular}{|c|c|c|c|}
\hline Category & & Frequency & Percentage \\
\hline \multirow{2}{*}{ Skip meal } & YES & 172 & 42.3 \\
\hline & NO & 235 & 57.7 \\
\hline \multirow{3}{*}{ Time of skip } & Some times & 98 & 56.98 \\
\hline & Fasting Day & 74 & 43.02 \\
\hline & at break Fast & 78 & 45.35 \\
\hline \multirow[t]{2}{*}{ When they skip } & at lunch & 8 & 3.49 \\
\hline & at dinner & 88 & 51.16 \\
\hline \multirow{6}{*}{ Reason for skip } & No food in the home & 11 & 6.39 \\
\hline & Fear of nausea and vomiting & 9 & 5.23 \\
\hline & Strong dislike & 39 & 22.67 \\
\hline & Abdominal discomfort & 38 & 22.09 \\
\hline & Fasting & 32 & 18.6 \\
\hline & Others & 43 & 25 \\
\hline
\end{tabular}

Alcohol and Coffee consumption of Pregnant Women

Concerning alcohol and coffee consumption; among 407 pregnant women 134 (32.9\%) drunk alcohol and 388 (95.3\%) drunk coffee.

\subsection{Family Planning and Antenatal Care}

Among 407 respondent 311 (76.4\%) attend health institution. From those who attend Health facility during pregnancy 311 (76.4\%) for Antenatal care service is 228 (73.31\%), 43 (13.83\%) for taking their family members, 33 (10.61\%) for medical treatment and 7 (2.25\%) sick during pregnancy [Figure 12].

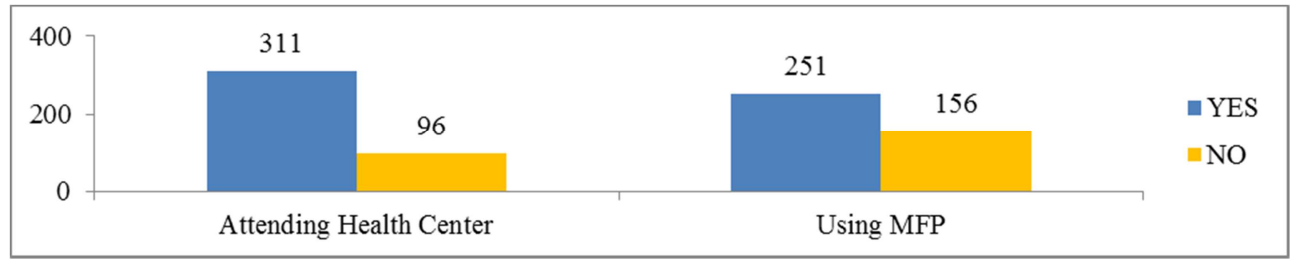

Figure 12. Attending Health center and Practicing previous MFP status of pregnant women in SendafaBeke town, Oromia Regional State, Ethiopia, June, 2019

\subsection{Pica Experience}

From 407 Study participants $104(25.6 \%)$ have practicing Pica Foods and ate $97(93.27 \%), 5(4.81 \%), 2$ (1.92\%), leftover food, Clay and soil respectively.

Multivariate analysis of factors associated with food taboo Variables having a $\mathrm{P} \leq 0.25$ in the bivariate analysis were fit into the final model after adjusting for potential confounders. After adjusting possible confounding factors in the multivariable logistic regression, educational status of mothers, occupation of husband, family size and eating habit like aversion and skipping meal have significantly associated with food taboo practice. Mothers who were unable to write and read 12.474 times more likely to practice food taboo than 
those who were college level and diploma group, [AOR and 95\% CI, $12.474(1.167,19.338)]$. Mothers husband those who were governments $98 \%$ less likely practice food taboo than Merchant mothers husband AOR and 95\% CI, 0.015 (0.001, 0.327). From study participants mothers who had $\geq 7$ family size were 2.877 more likely practice food taboo rather than 4-6 members of family size, AOR and 95\% CI, 2.877
(1.062, 34.025). Mothers who averted foods 44.237 more likely to be exposed to food taboo rather than those who not averted, AOR and 95\% CI, 44.237 (28.856, 51.003) and pregnant Women's who skipping meal 6.457 more likely to be exposed to food taboo rather than who not skip meal with Adjusted odds ratio of $6.457(1.584,26.324)$ at $95 \%$ CI [Table 7, and Table 8].

Table 7. Multiple Regression analysis of socio-demographic status versus Food taboo associated factors among pregnant women's in SendafaBeke town, June, 2019.

\begin{tabular}{|c|c|c|c|c|c|}
\hline \multirow{2}{*}{ Variables } & & \multicolumn{2}{|c|}{ Food Taboo } & \multirow{2}{*}{$\operatorname{COR}(95 \% \mathrm{CI})$} & \multirow{2}{*}{$\operatorname{AOR}(95 \% \mathrm{CI})$} \\
\hline & & Yes & No & & \\
\hline \multirow{5}{*}{ Age group (in years) } & $\leq 19$ & 11 & 3 & $0.341(0.070,1.654)$ & $0.949(0.033,27.315)$ \\
\hline & $20-24$ & 90 & 29 & $0.403(0.145,1.116)$ & $0.845(0.091,7.894)$ \\
\hline & $25-29$ & 61 & 92 & $1.885(0.704,5.045)$ & $0.489(0.076,3.157)$ \\
\hline & $30-34$ & 53 & 50 & $1.179(0.431,3.227)$ & $0.835(0.175,3.988)$ \\
\hline & $\geq 35$ & 10 & 8 & 0.800 & 1.000 \\
\hline \multirow{5}{*}{ Educational status } & unable to read and write & 46 & 15 & $2.515(1.231,5.137)$ & $12.474(1.167,19.338)$ \\
\hline & able to read and write & 50 & 41 & $2.108(1.072,4.146)$ & $1.124(1.105,7.674)$ \\
\hline & Primary [1-8] & 80 & 55 & $1.090(0.482,2.465)$ & $1.233(1.045,17.60)$ \\
\hline & Secondary [9-12] & 45 & 16 & $42.167(13.082,135.94)$ & $4.659(0.026,49.882)$ \\
\hline & College diploma and above & 4 & 55 & & \\
\hline \multirow{5}{*}{ Occupation of Husband } & Govt. employee & 77 & 80 & $0.708(0.342,1.466)$ & $0.267(0.036,1.995)$ \\
\hline & Merchant & 105 & 59 & $0.383(0.185,0.795)$ & $0.015(0.001,0.327)$ \\
\hline & Daily Laborer & 3 & 1 & $0.227(0.22,2.398)$ & $0.000(0.000)$, \\
\hline & Farmer & 22 & 18 & $0.558(0.226,1.379)$ & $1.297(0.198,8.508)$ \\
\hline & Others & 15 & 22 & & \\
\hline \multirow{3}{*}{ Family Size } & $1-3$ & 105 & 27 & $0.386(0.180,0.826)$ & $0.070(0.001,9.058)$ \\
\hline & $4-6$ & 96 & 139 & $2.172(1.096,4.304)$ & $2.877(1.062,34.025)$ \\
\hline & $\geq 7$ & 24 & 16 & & \\
\hline
\end{tabular}

Table 8. Multiple Regression analysis of eating behavior with Food taboos among pregnant women's in SendafaBeke town, June, 2019.

\begin{tabular}{|c|c|c|c|c|c|}
\hline \multirow{2}{*}{ Variables } & & \multicolumn{2}{|c|}{ Food Taboo } & \multirow{2}{*}{ COR $(95 \% C I)$} & \multirow{2}{*}{ AOR $(95 \% C I)$} \\
\hline & & Yes & No & & \\
\hline \multirow{2}{*}{ Aversion } & Yes & 205 & 47 & \multirow{2}{*}{$29.441(16.707,51.882)$} & \multirow{2}{*}{$44.237(28.856,51.003)$} \\
\hline & No & 20 & 137 & & \\
\hline \multirow{2}{*}{ Craving } & Yes & 151 & 59 & \multirow{2}{*}{$4.254(2.804,6.454)$} & \multirow{2}{*}{$1.411(0.219,9.108)$} \\
\hline & No & 74 & 123 & & \\
\hline \multirow{2}{*}{ Eat Vegetable and fruits } & Yes & 127 & 80 & \multirow{2}{*}{$0.605(0.408,0.897)$} & \multirow{2}{*}{$1.432(0.254,8.068)$} \\
\hline & No & 98 & 102 & & \\
\hline Additional meal & No & 141 & 144 & $0.443(0.283,0.693)$ & $0.767(0.144,4.076)$ \\
\hline \multirow{2}{*}{ Skipping Meal } & Yes & 109 & 63 & \multirow{2}{*}{$0.563(0.377,0.842)$} & \multirow{2}{*}{$6.457(1.584,26.324)$} \\
\hline & No & 116 & 119 & & \\
\hline \multirow{2}{*}{ Alcohol drink } & Yes & 96 & 38 & \multirow{2}{*}{$2.820(1.808,4.398)$} & \multirow{2}{*}{$8.421(0.992,71.364)$} \\
\hline & No & 129 & 144 & & \\
\hline
\end{tabular}

\subsection{Qualitative Study Result}

A total of 30 participants were selected in to 4 FGD. In the town, the research team conducted FGDs with all four groups of participants at four different Venues each lasting about an hour. I sought to find out the existence of food taboos and beliefs during pregnancy in SendafaBeke town identified the prohibited foods, how widespread is the practice and in depth explanation regarding reasons for the adherence by pregnant women.

All participants admitted knowing what food is and what it meant during pregnancy. The meaning of Food taboo as noted from participants across the all FGDs. Non-conclusive and divergent opinion were raised and entertained. From them, any foods that you are not supposed to eat or to drink during pregnancy. Foods when you eat can harm you or cause problems for the community. Some of pregnant mothers believe that it is an old story out that foods are taboos to eat. Totally and some pregnant women believe that there are taboos associated with pregnancy. The first category of taboo of pregnant mothers mentioned any white color, milk and milk products, Egg, linseed, and eating leafy vegetables.

Some of pregnant women believe that if pregnant women eats linseed, this will go to the abortion and if pregnant women eats leafy vegetables, this will go to the womb and attach to the baby's head causing particles to appear on the fetal head at delivery. Believes on weight gain during pregnancy because of these eating egg and milk and milk products during pregnancy affects for delivery it makes fat baby. Having too big baby is very dangerous to the life of the 
mother and the child during labor.

An FGD discussant pregnant mother in Sorrow face said;

........... Uff it is very risky if the abdomen of pregnant women gets too big, for example previously I deliver my first child through surgery because of big baby." So I often decrease food intake to limit or minimize the size of the baby.

\section{Discussion}

The study assessed common food taboos and misconceptions during pregnancy. More than half $(55.3 \%)$ of study participants not eat one food type during their pregnancy which is almost two times higher than the study conducted in Awabel district (27\%), and also higher than the study done in Shashamane district $(49.8 \%)$. But the current finding is lower than the study conducted in Hadiya zone $(65 \%)$, and $(70.1 \%)$ Tanzania $[14,11,17]$. The Possible difference to other regions may be because of cultural difference from the study area. Educational status and family size of women have showed significant association with food taboo with an adjusted odds ratio (AOR) of 12.474 (95\%CI $1.167,19.338)$ and $2.877 \quad(95 \% \mathrm{CI} \quad 1.062, \quad 34.025)$ respectively. In line with this, the qualitative result showed that those pregnant women unable to read and write by their educational status said that no problem to practicing taboo food.

Concerning diet changes during pregnancy, 172 (42.3\%) of all respondents have skipping eating which is almost similar to the study done in Sidama zone dale Woreda (36.5\%) [18]. Reasons given to avoid eating different items were:-food items make the pregnant women fat so that their birth will be making difficult labor which is similar to study conducted in Sudan [19]. The reason to this food taboos were plastered on the fetal head fear of abortion and fatty baby while born which was consistent with study done in Shashamane, and Hadiya town $[11,14]$.

This study revealed that, practicing Food taboo during pregnancy was found to have significant association with eating habit like aversion and skipping meal, occupation of husband, family size, and educational level of mothers. Besides, this study indicated that no use of additional meal was also a factor to food taboo during pregnancy. Therefore, providing nutrition education for women and their husband aimed at changing their attitudes towards food taboos and appropriate feeding practices is very important.

\section{Conclusions and Recommendations}

\subsection{Conclusion}

More than half $(55.3 \%)$ of women in the study area were obligated to avoid specific food items due to belief and traditional views during their pregnancy. Occupation of husband, educational level of mothers, no use of additional meal, Skipping meal and family size are statistically significant factors to food taboos during pregnancy. Hence, educating women and their husbands about the importance of food intake during pregnancy needs to be given due attention by all concerned bodies in the study area.

\subsection{Recommendations}

Healthcare workers in the study area should give nutritional education, and health promotion on appropriate feeding practices during pregnancy and harmful traditional practices.

Healthcare workers working in maternity unit of the study areas needs to encourage male (husband) involvement in maternal health care.

Local community and traditional birth attendants should encourage pregnant women 'eating up' during pregnancy and to provide supplementary food to poor women who cannot afford.

Furthermore, health policy makers should give due attention on harmful beliefs about food taboos during pregnancy and included it in health education program at each levels.

\section{Lists of Abbreviations}

\author{
ANC:-Antenatal Care \\ AOR:-Adjusted Odds Ratio \\ BSc:-Bachelor of Science \\ COR:-Crude Odds Ratio \\ FP:- Family Planning \\ HEW:-Health Extension Worker \\ HP:-Health Post \\ TM:-Traditional Medicine \\ WHO:-World Health Organization
}

\section{Declarations}

Ethics approval and consent to participate

Prior to starting the study, the final proposal was submitted to Debre Behran University Review Board for ethical approval. Then ethical clearance was taken from Debre Behran University. After permission was taken from Addis Ababa Zuria Zonal Health department. All participants have informed the right not to answer any questions or all questions if they want. Respect and full confidentiality of the study participant's response was granted since only the investigator had access to the data. Then information was collected after securing oral consent from study participant. Permission was first sought and obtained from the kebele administrate after explaining the purpose of the study.

\section{Availability of Data and Materials}

The finding of this study is generated from the data collected and analyzed based on stated methods and materials. The original data supporting this finding are available from the corresponding author on reasonable request. 


\section{Competing Interests}

The authors declare that they have no competing interests.

\section{Authors' Contributions}

RW participated in the design of the study, performed the data collection and the statistical analysis and served as the corresponding author of the manuscript. ET and ZK supervised the study, ensured quality of the data, assisted in the analysis and interpretation of the data. All authors read and approved the manuscript.

\section{Acknowledgements}

We are grateful to SendafaBeke Town health office staff and administrate for their unfailing support and guidance during the whole activities of this thesis. We also indebted for the study participants for their co-operation without which this study could not have been possible.

\section{References}

[1] Santos-Torres MI and Vásquez-Garibay E, Food Taboos Among Nursing Mothers Of Mexico; journal of health population and nutrition, 2003, 21 (2): 142-149.

[2] Alonso EB. The impact of culture, religion and traditional knowledge on food and nutrition security in developing countries March 2015.

[3] Assefa D, Wassie E, Getahun M, Berhaneselassie M, and Melaku A. Harmful Traditional Practices, Awassa college, Awassa, 2005.

[4] Corha In Collaboration With IFHP Comprehensive Reproductive Health And Gender A Training Reference Manual May, 2011.

[5] Perez GM and Garcia AP, Nutritional taboos among the fullas in upper River Region, The Gambia, The journal of Anthropology 2013.

[6] Zerfu et al. Dietary habits food taboos, and perceptions towards weight gain during pregnancy, journal of health, population and nutrition 2016, 35: 22.
[7] Demisse T. Muroki N and Kogi-makau W. Food taboos among Pregnant Women in Hadiya Zone, Ethiopia, Ethiopian journal of health development, 1998, 12.

[8] Mohamad M, and Yee Ling Ch. Food taboos of malay pregnant women attending antenatal check-up at the maternal health in kuala lumpur: Integrated Food, Nutrition and Metabolism, 2016.

[9] Central statistical Agency (Ethiopia) and ICF international: Ethiopia demographic and health survey Addis Ababa, Ethiopia, and Calverton Maryland, USA; Central statical Agency and ORC macro; 2014.

[10] C Rotimi et al. Nutritional Status in rural Nigeria; European journal of clinical nutrition 1999, 53, 734-39.

[11] McGuire and Pokin. Beating the Zero-sum game, women and Nutrition in the third world past. Food/Nutr. Bull., 1998; 11 (4) $38-63$

[12] Robert et al., Nutritional status in rural Nigeria: European Journal of clinical nutrition 2008; 53, 734-739.

[13] Anderson AS. Pregnancy as a time for dietary changes: Proc. Nut. Soc, 2001; 60 (6): 497-504.

[14] Dr. Bridget I. Feeding patterns of pregnant women in Delta State: Implication for nutrition education, Nigeria, 2010.

[15] Wen et al. Dietary Behaviors during pregnancy: findings from first-time mothers; International journal of behavioral nutrition and physical activity in southwest Sydney, 20107: 13.

[16] Man'ombe S. Pregnant Women As Beneficiaries Of The Vulnerable Group Feeding (Vgf) Programme In Mbire District Zimbabwe: An Assessment Of Nutritional Vulnerability; 2012.

[17] Zepro NB. Food taboos and misconceptions among pregnant women of Shashamene district, Ethiopia, Science Journal of Public health, 2012; 3, 410-46.

[18] Getnet W, Aycheh W, and Tessema T. Determinants of food taboos in the pregnant women of the Awabel district: Ethiopia, Advance in public health, 2018.

[19] Hassan Tahir HM, Elawad Mohammed Ahmed A, and Ahmed Ali Mohammed N. Food Taboos among Pregnant Women in Health Centers, Khartoum State- Sudan, 2016. Austin J Public Health Epidemiol. 2018; 5 (2): 1074. 